\title{
Externalities and Optimal Taxation
}

\author{
Helmuth Cremer \\ IDEI and GREMAQ \\ University of Toulouse \\ Toulouse, France
}

Firouz Gahvari

Department of Economics

University of Illinois at Urbana-Champaign

Champaign, IL 61820, USA

Norbert Ladoux

IDEI and GREMAQ

University of Toulouse

Toulouse, France

April 1997 


\begin{abstract}
This paper re-examines the optimal tax design problem (income and commodities) in the presence of externalities. The nature of the second-best, and the choice of the tax instruments, are motivated by the informational structure in the economy. The main results are: (i) environmental levies must generally consist of Pigouvian and nonPigouvian terms and be nonlinear; (ii) externalities do not affect commodity tax formulas (linear and nonlinear) for private goods; (iii) externalities do not affect the income tax structure if commodity taxes are nonlinear and affect it if commodity taxes are linear; and (iv) a general income tax plus strictly Pigouvian taxes are sufficient for efficient taxation if individuals of different types have identical marginal rates of substitution (at any given consumption bundle).

JEL classification: $\mathrm{H} 21 ; \mathrm{H} 23$
\end{abstract}

Keywords: optimal taxation; externalities; environmental levies; second-best; informational structure 


\section{Introduction}

The role of public policy in combating environmental problems is a hotly debated issue among economists and non-economists alike. The only consensus in academic circles is that if there is to be public intervention, it should be incentive-based rather than command and control. The most fully-developed incentive approach is the Pigouvian tax scheme. The idea is to levy a tax on an externality generating activity equal to its marginal social damage. This is a first-best remedy which, in the absence of other distortions in the economy (including distortionary taxes), moves the competitive equilibrium of the economy to its Pareto-efficient frontier. In a second-best environment, however, this prescription must be modified.

This problem was originally studied by Sandmo (1975), and more recently by Bovenberg and van der Ploeg (1994). ${ }^{1}$ The main result that has emerged from this literature is what Sandmo dubbed the "additivity property" where the presence of externality only alters the tax formula for the externality generating good, leaving the tax formulas for other goods unaffected. Dixit (1985) has referred to Sandmo's result as an instance of the more general "principle of targeting". The idea is that one should best counter a distortion by the tax instrument that acts on it directly. Bovenberg and van der Ploeg (1994) also emphasize this principle in their finding that the formula for the labor income tax must also remain unaffected.

While this literature has been motivated by the recognition that second-best considerations may alter the characterization of optimal taxes, its treatment of the second-best has been quite arbitrary. In particular, it has assumed that the feasible tax instruments, including the income tax, are all linear. This is important. Atkinson and Stiglitz (1976) have taught us that the properties of optimal tax schemes depend crucially on the instru-

\footnotetext{
${ }^{1}$ Bovenberg and van der Ploeg (1994) examine a number of other issues including the impact of externalities on employment as well as on the optimal level and composition of public spending. These latter issues are not the concern of this paper. Nor are we concerned with the taxation of intermediate inputs as in Bovenberg and Goulder (1996).
} 
ments allowed. The aim of the current paper is to re-examine the optimal tax problem in the presence of externalities while motivating the choice of the tax instruments by the informational structure in the economy.

We pose two basic questions. The first question concerns the nature of the optimal tax on an externality generating good when first-best taxes are not available. Under what circumstances does the tax differ from the Pigouvian tax? What factors determine the sign and the magnitude of this difference? The second question inquires into the properties of second-best taxes on "private" good and income in the presence of externalities. In particular, what are the interconnections between environmental levies and traditional tax instruments? The answers to these questions crucially depend on the delineation of the second-best itself; namely the extent and the nature of the constraints that prevent imposition of first-best taxes. We will consider two such specifications in this paper based on the informational constraints the government faces.

We model an economy with $n$ private goods, labor supply and an externality generating good. The externality is created by the total consumption of the good in question. Many environmental problems are due to this type of externality; greenhouse effect is an example. ${ }^{2}$ There are $H$ types of persons in the economy who may differ in two dimensions: earning abilities and tastes. This is a generalization of Stiglitz's $(1982,1987)$ two-group optimal tax model when individuals differ only in earning abilities. In the tradition of the optimal tax literature, we assume that the types are not publicly observable. This makes first-best tax instruments unavailable. However, personal income levels are observable so that non-linear income taxation is feasible.

In the first part of the paper (Section 3), we follow the implicit assumption of Stiglitz that personal consumption levels are publicly observable. This assumption allows commodity taxes to be levied in a non-linear fashion. We then characterize the properties of second-best taxes in this environment. Answering our first question, we

\footnotetext{
${ }^{2}$ Meade (1952) has termed such externalities "atmosphere" externalities. For the issues pertaining to greenhouse effect and global warming, see Dornbusch and Poterba (1991).
} 
show that the tax on the externality generating good is non-linear, with a formula that contains both Pigouvian and non-Pigouvian elements. Recall that the first-best tax is linear with an identical rate for everybody; it is also strictly Pigouvian in nature. Answering our second question, we show that the presence of the externality does not affect the formulas determining the optimal commodity taxes on private goods and income taxes. This finding is in agreement with Sandmo's additivity property.

We next extend Atkinson and Stiglitz's (1976) famous result [and Mirrlees's (1976) generalization of it] on the usefulness of commodity taxes in the presence of a general income tax, to a setting with externality. We prove that when individuals of different types have identical marginal rates of substitution (at any given consumption bundle), differential commodity taxation is not called for and the optimal tax on the externality generating good is strictly Pigouvian. Moreover, for a two-group case, we find conditions under which the externality tax must be greater or smaller than the Pigouvian tax.

In the second part of the paper (Sections 4-6), we drop the assumption of public observability of personal consumption levels and examine the optimal tax problem anew. This is motivated by the nature of information typically available to tax administrations. While total purchases of a commodity are generally observed, individual consumption levels are often private information. As a rule, the tax administration does not know the identity of who buys how much of what good. Lack of public observability of personal purchases precludes the levying of non-linear commodity taxes. The best that the government can do is to impose (possibly differential) linear commodity taxes.

Given this informational structure, we characterize Pareto-efficient allocations that are constrained, in addition to resource balance and the standard self-selection constraints, by the linearity of commodity taxes. To do this, we derive an optimal revelation mechanism. For our purpose, a mechanism consists of a set of type-specific before-tax and after-tax incomes and a vector of commodity tax rates (same for everyone). This procedure determines the commodity tax rates right from the outset. A complete solu- 
tion to the optimal tax problem per-se then requires only the design of an implementing income tax function.

We give a characterization for optimal commodity taxes and examine the properties of the marginal income tax rate. Answering our first question anew, we show that the optimal externality tax continues to consist of both Pigouvian and non-Pigouvian elements. Regarding our second question, we show that while the tax formulas for private goods remain unaffected, the structure of income taxation changes. That is, the presence of externality has different implications for commodity and income taxation. This indicates that Sandmo's additivity property, and Dixit's Principle of targeting, is not a universal property. It depends on the nature of the tax instruments that are available (i.e. if linear or nonlinear) for direct as against indirect intervention.

Independently from the externality issue, the paper also makes a methodological contribution to the optimal tax literature. Building on Cremer et al. (1996), it studies Pareto-efficient tax structures in a model with $H$ types of individuals and two unobservable characteristics. It derives general results on the properties of income and commodity taxes for two informational structures: public observability of consumption levels at (i) personal and (ii) aggregate levels.

\section{The model}

Consider an economy consisting of a continuum of individuals who can be grouped into $H$ different and finite types. The types may differ in two characteristics: earning abilities and tastes. Each person, regardless of his type, is endowed with one unit of time. He has preferences over labor supply and $n+1$ consumer goods. All goods are produced by a linear technology subject to constant returns to scale. The $(n+1)$ th good creates a negative consumption externality; the first $n$ goods (labelled private goods) do not. The externality is created by the total consumption of $y$. This is the type Meade (1952) has termed "atmosphere" externalities. Normalize the population size at one and denote 
the proportion of persons of type $j$ in the population by $\pi^{j}$. Further denote the vector of private goods by $\underline{x}=\left(x_{1}, x_{2}, \ldots, x_{n}\right)$, the externality generating good by $y$, the labor supply by $L$, and normalize producer prices of all consumer goods at one.

A person of type $j$ has $w^{j}$ as his wage and

$$
\mho^{j}=\boldsymbol{U}\left(\underline{x}, y, L, \theta^{j}\right)-\phi\left(\sum_{j=1}^{H} \pi^{j} y^{j}\right), \quad j=1,2, \ldots, H,
$$

as his utility function, where $\theta^{j}$ is a "taste" parameter. Note that while type $j$ differs from type $k(k \neq j)$, it is possible that $w^{j}=w^{k}$ or $\theta^{j}=\theta^{k}$. In words, we do not rule out the case where two types differ in one of the characteristics only. This is a very general specification assuming no particular correlation between the values of $\theta^{j}$ and $w^{j} .^{3}$

We assume that $\boldsymbol{U}$ is strictly quasi-concave, twice continuously differentiable, strictly increasing in $\underline{x}$ and $y$, and strictly decreasing in $L$; while $\phi$ is convex and increasing. The following points must be noted regarding the specification of preferences in (1). First, For the purpose of exposition, we shall take the externality to be detrimental. Thus $\phi($.$) appears in (1) with a negative sign. Second, the seemingly equal weight assigned$ to $\phi($.$) is only in appearance; it imposes no restrictions. Since \boldsymbol{U}$ depends on $\theta^{j}$, this is a matter of normalization. ${ }^{4}$ Third, allowing $\phi($.$) to also depend on \theta^{j}$ will entail only a slight modification in the expressions derived below; it is of no consequence. Fourth, the main assumption is that of separability of preferences between consumption goods and the externality. This is adopted for ease of exposition; it does not change the main conclusions of the paper.

In the tradition of the optimal income tax literature, we assume that an individual's type and labor input is not observable by the government. His before-tax income, $I=w L$, on the other hand, is. This rules out first-best taxation of types as a policy

\footnotetext{
${ }^{3}$ Specifically, $\theta^{j}$ and $w^{j}$ take $M(\leq H)$ and $N(\leq H)$ distinct values, resulting in $H(\leq M . N)$ different types in the economy.

${ }^{4}$ For any non-unitary weight on $\phi($.$) , one can divide \mho^{j}$ by that weight and return to the specification in (1).
} 
instrument while allowing non-linear taxation of incomes. It is then convenient to introduce a type-specific utility function describing preferences over $x_{i}$ 's, $y$ and $I$ :

$$
\boldsymbol{u}^{j}(\underline{x}, y, I) \equiv \boldsymbol{U}\left(\underline{x}, y, \frac{I}{w^{j}}, \theta^{j}\right),
$$

where $w^{j}$ is the wage of an individual of type $j$.

Given this framework, we set out to answer the two basic questions we posed in the Introduction. To do this, we specify two informational structures for the government; each delineating a different second-best environment.

\section{Observable individual purchases}

Suppose that in addition to before-tax incomes, $I^{j}$ 's, the government could also observe individual purchases, $x_{i}^{j}$ 's and $y^{j}$ 's, so that nonlinear commodity taxes are feasible. Tax structures will then be constrained by self-selection only. This is the framework used by Stiglitz $(1982,1987)$ to discuss Pareto-efficient self-selection tax structures. Of course, in his set-up, there is no externality. Moreover, he does not allow for taste differentiation and considers only two groups of persons who differ in earning abilities.

To derive the tax structure, we consider the standard equivalent problem of the government first choosing optimal allocations subject to resource balance, self-selection and the relevant non-negativity constraints. Having derived the optimal allocation, we then describe the tax structure that can implement it.

\subsection{Pareto-efficient allocations}

Let $\bar{R}$ denote the government's revenue requirement. Denote the utility level of a $j$-type individual by $u^{j}$ when he chooses the allocation intended for him, and by $u^{j k}$ when he chooses a $k$-type person's bundle. Thus denote

$$
\begin{aligned}
u^{j} & =\boldsymbol{u}^{j}\left(\underline{x}^{j}, y^{j}, I^{j}\right), \\
u^{j k} & =\boldsymbol{u}^{j}\left(\underline{x}^{k}, y^{k}, I^{k}\right) .
\end{aligned}
$$


One can then describe the set of Pareto-efficient allocations as follows. Maximize

$$
\sum_{j=1}^{H} \gamma^{j} \mho^{j}
$$

with respect to $\underline{x}^{j}, y^{j}$ and $I^{j}$; subject to the resource constraint

$$
\sum_{j=1}^{H} \pi^{j}\left(I^{j}-\sum_{i} x_{i}^{j}-y^{j}\right) \geq \bar{R},
$$

and the self-selection constraints

$$
u^{j} \geq u^{j k}, \quad j \neq k ; j, k=1,2, \ldots, H
$$

where $\gamma^{j}$ 's are positive constants with the normalization $\sum_{j=1}^{H} \gamma^{j}=1$.

Denote the Lagrangian expression by $\mathcal{L}$, and the (non-negative) Lagrangian multipliers associated with the resource constraint (5) by $\mu$, and with the self-selection constraints (6) by $\lambda^{j k}$. One can write ${ }^{5}$

$$
\begin{aligned}
\mathcal{L}=\sum_{j} \gamma^{j} \mho^{j} & +\mu\left[\sum_{j} \pi^{j}\left(I^{j}-\sum_{i} x_{i}^{j}-y^{j}\right)-\bar{R}\right] \\
& +\sum_{j} \sum_{k \neq j} \lambda^{j k}\left(u^{j}-u^{j k}\right) .
\end{aligned}
$$

Substitute for $\mho^{j}$ from (1) into (7), use $\sum_{j} \gamma^{j}=1$ and rearrange the terms to rewrite the Lagrangian expression as

$$
\begin{aligned}
\mathcal{L} & =\sum_{j}\left(\gamma^{j}+\sum_{k \neq j} \lambda^{j k}\right) u^{j}-\phi\left(\sum_{j} \pi^{j} y^{j}\right) \\
& +\mu\left[\sum_{j} \pi^{j}\left(I^{j}-\sum_{i} x_{i}^{j}-y^{j}\right)-\bar{R}\right]-\sum_{j} \sum_{k \neq j} \lambda^{j k} u^{j k} .
\end{aligned}
$$

This yields the following first-order conditions for $j=1,2, \ldots, H$, and $i=1,2, \ldots, n$.

$$
\frac{\partial \mathcal{L}}{\partial x_{i}^{j}}=\left(\gamma^{j}+\sum_{k \neq j} \lambda^{j k}\right) u_{i}^{j}-\mu \pi^{j}-\sum_{k \neq j} \lambda^{k j} u_{i}^{k j}=0,
$$

\footnotetext{
${ }^{5}$ To simplify notation, we use $\sum_{j}$ for $\sum_{j=1}^{H}$ and $\sum_{k \neq j}$ for $\sum_{\substack{k=1 \\ k \neq j}}^{H}$ throughout the paper.
} 


$$
\begin{aligned}
& \frac{\partial \mathcal{L}}{\partial y^{j}}=\left(\gamma^{j}+\sum_{k \neq j} \lambda^{j k}\right) u_{y}^{j}-\pi^{j} \phi^{\prime}-\mu \pi^{j}-\sum_{k \neq j} \lambda^{k j} u_{y}^{k j}=0, \\
& \frac{\partial \mathcal{L}}{\partial I^{j}}=\left(\gamma^{j}+\sum_{k \neq j} \lambda^{j k}\right) u_{I}^{j}+\mu \pi^{j}-\sum_{k \neq j} \lambda^{k j} u_{I}^{k j}=0
\end{aligned}
$$

where a subscript on $u$ denotes a partial derivative. Note that the calculation of the derivatives of $\sum_{j} \sum_{k \neq j} \lambda^{j k} u^{j k}$ results in the transposition of their $j$ and $k$ indices. Manipulating first-order conditions (9a)-(9c), one can derive the following equations for $j=1,2, \ldots, H$, and $i, s=1,2, \ldots, n$.

$$
\begin{aligned}
\frac{u_{y}^{j}}{u_{i}^{j}} & =\frac{1+\phi^{\prime} / \mu+\sum_{k \neq j} \lambda^{k j} u_{y}^{k j} / \mu \pi^{j}}{1+\sum_{k \neq j} \lambda^{k j} u_{i}^{k j} / \mu \pi^{j}}, \\
\frac{u_{s}^{j}}{u_{i}^{j}} & =\frac{1+\sum_{k \neq j} \lambda^{k j} u_{s}^{k j} / \mu \pi^{j}}{1+\sum_{k \neq j} \lambda^{k j} u_{i}^{k j} / \mu \pi^{j}}, \\
-\frac{u_{I}^{j}}{u_{i}^{j}} & =\frac{1-\sum_{k \neq j} \lambda^{k j} u_{I}^{k j} / \mu \pi^{j}}{1+\sum_{k \neq j} \lambda^{k j} u_{i}^{k j} / \mu \pi^{j}} .
\end{aligned}
$$

The system of equations (10a)-(10c) characterizes Pareto-efficient allocations constrained by self-selection. Note that these conditions, and our specification, are quite general. In particular, they are not based on the "single-crossing" property. The self-selection constraints associated with a $j$-type mimicking a $k$-type and a $k$-type mimicking a $j$-type can simultaneously bind; and one can also have "bunching".

The left-hand sides of (10a)-(10c) are the familiar marginal rates of substitution between different goods. ${ }^{6}$ When individuals do their purchases in the market, they set these rates equal to the relative prices they face. This observation allows us to examine the properties of the tax function which can implement these allocations.

\footnotetext{
${ }^{6}$ That $u_{y}^{j} / u_{i}^{j}$ is the relevant marginal rate of substitution follows because, when optimizing with respect to $y^{j}$, a $j$-type person treats $\sum_{j} \pi^{j} y^{j}$ as given. This is due to the continuum of individuals set-up of the paper. As far as $-u_{I}^{j} / u_{i}^{j}$ is concerned, one may think of it as a $j$-type person's marginal rate of substitution between the gross of tax income and good $i$, as well as $\left(1 / w^{j}\right)$ times his marginal rate of substitution between leisure and $\operatorname{good} i$.
} 


\subsection{First-best taxation}

It will be instructive to start by examining the nature of first-best taxes. Our characterization of the second-best above allows us to do this most simply. At first-best allocations, self-selection is not a constraint on the government's problem. Thus set $\lambda^{k j}=0$ in equations (10a)-(10c). This yields

$$
\begin{aligned}
\frac{u_{y}^{j}}{u_{i}^{j}} & =1+\frac{\phi^{\prime}}{\mu}, \\
\frac{u_{s}^{j}}{u_{i}^{j}} & =1, \\
-\frac{u_{I}^{j}}{u_{i}^{j}} & =1 .
\end{aligned}
$$

Equations (11a)-(11c) tell us that the implementation of first-best outcome requires, as expected, no distortionary taxes on $x_{i}^{j}$, s and $I^{j}$ but a tax on $y^{j}(j=1,2, \ldots, H)$ equal to $\phi^{\prime} / \mu$. Note that the required tax rate on $y$ is the same for everyone and equal to the marginal social damage associated with any person's purchase of a last unit of $y$. This is the case because the nature of the externality is such that only aggregate size of $y$ $\left(=\sum_{j} \pi^{j} y^{j}\right)$ matters.

In what follows, for concreteness, we shall follow the following terminology.

Definition 1 A tax is called (i) Pigouvian if it is equal to $\phi^{\prime} / \mu$ (the marginal social damage of $\sum_{j} \pi^{j} y^{j}$ ) and (ii) non-Pigouvian if $\phi^{\prime} / \mu$ does not appear in the expressions that determine it.

\subsection{Second-best taxation}

When at least one of the self-selection constraints in (6) is binding, the optimal allocation is no longer first-best. The allocations to be implemented are thus characterized by (10a)-(10c) [rather than by (11a)-(11c)]. Consider (10a); the equation characterizing the second-best tax on $y$. It shows that any groups of persons, $l$, for whom $\lambda^{k l} \neq 0$, for 
some $k$, must pay a tax on $y$ which will generally be different from $\phi^{\prime} / \mu$. In a sense, this is to be expected. Our setting also requires that the tax treatment of private goods to depart from first-best (i.e. they should be taxed rather than go tax free). It is thus only logical that the same principle should hold for the tax treatment of non-private goods.

One can nevertheless make three important observations regarding the tax treatment of $y$. First, the formula characterizing the marginal tax on $y$ contains Pigouvian and non-Pigouvian terms. Second, the non-Pigouvian term is the same as in the formula for the optimal tax on private goods. That is, the formulas for taxation of private and non-private goods differ only in that the latter entails a Pigouvian term. Third, as will be shown below, unless one places certain restrictions on preferences, different types of persons face different marginal tax rates on $y$ depending on which self-selection constraints bind. Put another way, the optimal tax on $y$ is non-linear. Contrast these with first-best taxes where everyone, regardless of his type, faces a constant marginal tax rate equal to $\phi^{\prime} / \mu$. While there may be some types of persons who would pay the Pigouvian tax here, this will not be the case for all types. ${ }^{7}$

Next, turn to (10b)-(10c), the equations characterizing optimal taxes on private goods and income. These equations do not contain any externality terms; they are identical to the equations governing optimal second-best taxes in the absence of the externality. In this sense, one may say that the presence of the externality does not change the structure of second-best taxes on private goods and income. ${ }^{8}$

This last result was originally derived by Sandmo (1975) in a setting with proportional income and commodity taxes. He dubbed it the "additivity property" whereby "the marginal social damage of commodity $m$ enters the tax formula for that commodity additively, and does not enter the tax formulas for other commodities..." (p. 92). Later,

\footnotetext{
${ }^{7}$ The assumption that $x_{i}^{j}$ 's and $y^{j}$ are publicly observable implies that the government will have no difficulty in levying non-linear taxes. Indeed, the tax imposed on a given commodity can even depend on the purchaser's consumption of other commodities as well as on his income.

${ }^{8}$ Of course, same formula does not mean same level.
} 
Dixit (1985) referred to Sandmo's result as an instance of the more general "principle of targeting". As he put it, this principle states that "a distortion is best countered ... by a tax instrument that acts directly on the relevant margin." (p. 314). Bovenberg and van der Ploeg (1994) emphasize this principle in their finding, again assuming linear taxes, that the formula for the labor income tax must also remain unaffected. While our result here is in accordance with this property, we will see in subsequent sections that this may not always be the case.

Finally, for the purpose of later comparisons, consider the famous "no distortion at the top" result of the optimal tax theory. [See, e.g., Mirrlees (1971) and Seade (1977)]. This result continues to hold here. To see this, assume there exists a category of persons, $h$, whom nobody mimics at equilibrium. ${ }^{9}$ This is the counterpart in our multicharacteristic setting to the "top" individual in standard optimal income tax models. No incentive constraint is thus binding towards this category, so that $\lambda^{k h}=0$ for all $k(\neq h)$. It then immediately follows from (10b)-(10c) that no distortionary taxes must be levied on these persons' income and consumption of private goods. Moreover, from (10a), they should face a simple Pigouvian tax on their consumption of $y$. The intuition is just as in the traditional case. Since at equilibrium nobody wants to mimic an $h$-type person, there is no advantage in distorting such a person's consumption bundle from the first-best.

\subsubsection{Separable preferences and homogeneous tastes}

An interesting case arises when individuals of different types have identical marginal rates of substitution between any given two consumer goods (at the same consumption bundle). That is, assume the following conditions hold everywhere.

$$
\frac{u_{y}^{k j}}{u_{i}^{k j}}=\frac{u_{y}^{j}}{u_{i}^{j}} \quad j=1,2, \ldots, H, \text { and } i=1,2, \ldots, n,
$$

\footnotetext{
${ }^{9}$ When individuals differ in both earning abilities and tastes, this category may or may not exist.
} 


$$
\frac{u_{s}^{k j}}{u_{i}^{k j}}=\frac{u_{s}^{j}}{u_{i}^{j}} \quad i, s=1,2, \ldots, n .
$$

Conditions (12a)-(12b) are guaranteed when (i) preferences are separable between labor supply and other goods (as in Atkinson and Stiglitz) and (ii) tastes are homogeneous in the sense that the marginal rates of substitution of different persons (for non-leisure goods) are independent of the taste parameter $\theta^{j} \cdot{ }^{10}$ Given (12a)-(12b), one can easily show that equations (10a)-(10b) will reduce to (11a)-(11b). This implies that the structure of commodity taxes that implement the second-best allocations characterized by (10a)-(10c) remains precisely the same as that in the first-best. That is, there should be no taxes on $x_{i}^{j}$ 's but that all types must face the same Pigouvian tax on $y^{j}$. [These preferences have no implications for the structure of income taxes].

This result is reminiscent of Atkinson and Stiglitz's (1976) seminal result, and its generalization by Mirrlees (1976), concerning the usefulness of commodity taxes in the presence of a general income tax. In the absence of externality, when conditions (12a)(12b) hold, commodity taxes are not needed because they cannot be used as a basis for separation between individuals of different types. [See Stiglitz $(1982,1987)$ ]. That is, they cannot be used to relax otherwise binding self-selection constraints and effect a Pareto improvement. The same intuition applies to our setting when there is an externality present. Consumption of private goods must not be distorted, and the only adjustment needed for the externality generating good is the familiar Pigouvian one.

When conditions (12a)-(12b) do not hold, the second-best tax on $y$ departs from the Pigouvian tax and differs across types. The precise characterization of such departures for all individual types is an interesting but complicated question. The next subsection attempts to shed some light on this issue by considering a special case.

\footnotetext{
${ }^{10}$ Preferences satisfying these properties are represented by

$$
U\left(\underline{x}, y, L, \theta^{j}\right)=\Omega\left(\theta^{j} F(\underline{x}, y), L\right) .
$$

Atkinson and Stiglitz (1976) assume identical preferences at the outset. Mirrlees (1976) discusses taste differentiation.
} 


\subsubsection{A two-group illustration}

Assume there are two types of skills and two types of tastes. Each skill level is associated with a particular taste parameter so that there will be only two types of persons. ${ }^{11}$ Individuals indexed by $l$ are less skilled earning a lower wage than those indexed by $h$. Further assume that preferences are separable in $\underline{x}, y$ and $L$. Specifically, assume

$$
u^{j}(\underline{x}, y, I)=f(\underline{x})+\theta^{j} h(y)+\varphi\left(\frac{I}{w^{j}}\right) .
$$

Consider the Pareto-efficient allocations at which only the downward self-selection constraint is binding. ${ }^{12}$ This is when redistribution is from the high- to low-ability persons. Setting $\lambda^{h l}>0$ and $\lambda^{l h}=0$ in (10b), it immediately follows that

$$
\frac{u_{s}^{h}}{u_{i}^{h}}=\frac{u_{s}^{l}}{u_{i}^{l}}=1
$$

This tells us that the tax rates on $x_{i}^{j}$, s $(j=h, l)$ must be uniform. This result is not surprising in light of our result in Subsection 3.3.1 and the structure of preferences (separability in $L$ and other goods and the uniformity of tastes for $\underline{x}$ ). Given one extra degree of freedom in setting the tax rates for every individual, this tax can then be set at zero.

Turning to the taxation of $y$, from (10a), one has: $u_{y}^{h} / u_{i}^{h}=1+\phi^{\prime} / \mu$. This indicates a Pigouvian tax for the $h$-type. On the other hand, (10a) also yields

$$
\frac{u_{y}^{l}}{u_{i}^{l}}=1+\frac{\phi^{\prime}}{\mu}+\frac{\lambda^{h l} h^{\prime}\left(y^{l}\right)}{\mu \pi^{l}}\left(\theta^{h}-\theta^{l}\right),
$$

which immediately implies

$$
\begin{aligned}
& \frac{u_{y}^{l}}{u_{i}^{l}}>1+\frac{\phi^{\prime}}{\mu}, \quad \text { if } \quad \theta^{l}<\theta^{h}, \\
& \frac{u_{y}^{l}}{u_{i}^{l}}<1+\frac{\phi^{\prime}}{\mu}, \quad \text { if } \quad \theta^{l}>\theta^{h} .
\end{aligned}
$$

\footnotetext{
${ }^{11}$ Without this assumption, there can be as many as four individual types.

${ }^{12}$ As in footnote 8 above, with multi-dimensional heterogeneity, such a regime may or may not exist.
} 
Equations (16a)-(16b) show that $l$-type persons must face a tax on their consumption of $y$ that may be greater as well as smaller than the Pigouvian tax. The intuition behind these equations must be seen in the ability of the government to use commodity taxes to relax an otherwise binding self-selection constraint. In either case, the idea is to make the $l$-type's bundle less appealing to an $h$-type, making the $h$-type more reluctant to want to mimic the $l$-type. For example, if $\theta^{h}>\theta^{l}$, the (mimicking) $h$-type values $y$ more than the $l$-type. The tax on $y$ will then affect the rich more than the poor.

The following proposition summarizes the main results of this section.

Proposition 1 Assume personal consumption levels are publicly observable. Then

(i) if individuals of different types have identical marginal rates of substitution (at any given consumption bundle), (a) the optimal second-best tax on the externality generating good, $y$, is Pigouvian (and thus the same for everyone), and (b) private goods must not be taxed;

(ii) with non-identical marginal rates of substitution, the optimal second-best tax on $y$ is nonlinear with a formula that contains both Pigouvian and non-Pigouvian terms;

(iii) the presence of the externality does not affect the formulas determining optimal commodity taxes on private goods and optimal income taxes;

(iv) with two types of persons, preferences that are represented by (13), $\lambda^{h l}>0$ and $\lambda^{\text {lh }}=0$, (a) high-ability persons should face a Pigouvian tax on their consumption of $y$, while low-ability persons must face a tax greater (smaller) than Pigouvian if they, in comparison to h-types and for the same consumption bundle, have a "lower" ("higher") marginal rate of substitution of $y$ for $x_{i}$, and (b) private goods must go untaxed.

\section{Observable aggregate purchases}

The results of the previous section rests crucially on the assumption that personal consumption levels are publicly observable. This assumption allows the government to 
levy non-linear commodity taxes. Moreover, as we saw in Section 3, such taxes are in fact necessary to implement Pareto-efficient allocations (constrained by self-selection). However, this assumption is rather hard to justify on informational grounds. It is more realistic to assume that the tax administration has information on anonymous transactions (i.e. aggregate sales of a commodity rather than who purchases how much). This is the standard assumption in the literature, so much so that it has been used as part of very definition of indirect taxes. [See, e.g., Atkinson and Stiglitz (1980, p. 427)]. Under this circumstance, non-linear commodity taxes are not feasible. If , for instance, the tax rate is linked to the quantity purchases, the buyer can avoid higher taxes by splitting the transactions. As a rule, only linear commodity taxes are available.

It should be pointed out that the informational requirement for non-linear commodity taxation is much more stringent than for non-linear income taxation. The latter type of taxes require information on each person's aggregate expenditure (or equivalently income) only. Non-linear commodity taxes, on the other hand, require information on each person's expenditure on every single good. The linearity of commodity taxes is thus a direct implication of the informational structure typically available in the economy.

Given this informational structure, one may proceed to characterize Pareto-efficient allocations that are constrained, in addition to resource balance, not only by the standard self-selection constraints but also by the linearity of commodity taxes. To do this, we derive an optimal revelation mechanism. For our purpose, a mechanism consists of a set of type-specific before-tax incomes, $I^{j}$ 's, aggregate expenditures on private goods, $c^{j}$ 's, and a vector of commodity tax rates (same for everyone). This procedure determines the commodity tax rates, $\underline{t}=\left(t_{1}, t_{2}, \ldots, t_{n}\right)$ and $t_{y}$, right from the outset. A complete solution to the optimal tax problem per-se then requires only the design of a general income tax function. Note that instead of commodity taxes, the mechanism may equivalently specify the consumer prices of $\underline{x}$ and $y$, denoted by $\underline{p}=\left(p_{1}, p_{2}, \ldots, p_{n}\right)$ 
and $q$, where $p_{i}=1+t_{i}(i=1,2, \ldots, n)$ and $q=1+t_{y} \cdot{ }^{13}$

To proceed further, it is necessary to consider the optimization problem of an individual for a given mechanism $(\underline{p}, q, c, I)$. This is necessitated by the fact that the mechanism determines personal consumption levels (on private goods) only indirectly, namely through prices. The mechanism assigns $\left(\underline{p}, q, c^{j}, I^{j}\right)$ to an individual who reports type $j$. The consumer then allocates $c^{j}$ between the produced goods, $\underline{x}$ and $y$.

Formally, given any vector $(\underline{p}, q, c, I)$, an individual of type $j$ solves

$$
\begin{gathered}
\max _{\underline{x}, y} \boldsymbol{u}^{j}(\underline{x}, y, I) \\
\text { subject to } \sum_{i=1}^{n} p_{i} x_{i}+q y=c .
\end{gathered}
$$

The resulting demand functions are denoted by $\boldsymbol{x}_{i}^{j}(\underline{p}, q, c, I)$ and $\boldsymbol{y}^{j}(\underline{p}, q, c, I)$, and the indirect utility function by

$$
\boldsymbol{v}^{j}(\underline{p}, q, c, I) \equiv \boldsymbol{u}^{j}\left(\underline{x^{j}}(\underline{p}, q, c, I), y^{j}(\underline{p}, q, c, I), I\right)
$$

For ease of notation, we define

$$
\begin{aligned}
x_{i}^{j} & =\boldsymbol{x}_{i}^{j}\left(\underline{p}, q, c^{j}, I^{j}\right), \\
y^{j} & =\boldsymbol{y}^{j}\left(\underline{p}, q, c^{j}, I^{j}\right), \\
v^{j} & =\boldsymbol{v}^{j}\left(\underline{p}, q, c^{j}, I^{j}\right), \\
x_{i}^{j k} & =\boldsymbol{x}_{i}^{j}\left(\underline{p}, q, c^{k}, I^{k}\right), \\
y^{j k} & =\boldsymbol{y}^{j}\left(\underline{p}, q, c^{k}, I^{k}\right), \\
v^{j k} & =\boldsymbol{v}^{j}\left(\underline{p}, q, c^{k}, I^{k}\right), \\
V^{j} & =v^{j}-\phi\left(\sum_{j} \pi^{j} y^{j}\right) .
\end{aligned}
$$

\footnotetext{
${ }^{13}$ Strictly speaking, this procedure does not characterize "allocations" as such; the optimization is over a mix of quantities and prices. However, given the commodity prices, utility maximizing individuals would choose the quantities themselves. We can thus think of the procedure as indirectly determining the final allocations.
} 


\subsection{Pareto-efficient (constrained) allocations}

Constrained Pareto-efficient "allocations" can be described as follows. Maximize

$$
\sum_{j} \gamma^{j} V^{j}
$$

with respect to $p_{2}, p_{3}, \ldots, p_{n}, q, c^{j}$ and $I^{j} ;^{14}$ subject to the resource constraint

$$
\sum_{j} \pi^{j}\left[\left(I^{j}-c^{j}\right)+\sum_{s=2}^{n}\left(p_{s}-1\right) x_{s}^{j}+(q-1) y^{j}\right] \geq \bar{R}
$$

and the self-selection constraints

$$
v^{j} \geq v^{j k}, \quad j, k=1,2, \ldots, H
$$

As in Section 3, denote the Lagrangian expression by $\mathcal{L}$, and the Lagrangian multipliers associated with the resource constraint (20) by $\mu$, and with the self-selection constraints (21) by $\lambda^{j k}$. We have

$$
\begin{aligned}
\mathcal{L}=\sum_{j} \gamma^{j} V^{j} & +\mu\left\{\sum_{j} \pi^{j}\left[\left(I^{j}-c^{j}\right)+\sum_{s=2}^{n}\left(p_{s}-1\right) x_{s}^{j}+(q-1) y^{j}\right]-\bar{R}\right\} \\
& +\sum_{j} \sum_{k \neq j} \lambda^{j k}\left(v^{j}-v^{j k}\right) .
\end{aligned}
$$

Substituting for $V^{j}$ from (18g) into (22) and rearranging the terms, one may usefully rewrite the Lagrangian expression as

$$
\begin{aligned}
\mathcal{L} & =\sum_{j}\left(\gamma^{j}+\sum_{k \neq j} \lambda^{j k}\right) v^{j}-\phi\left(\sum_{j} \pi^{j} y^{j}\right)+\mu\left\{\sum _ { j } \pi ^ { j } \left[\left(I^{j}-c^{j}\right)\right.\right. \\
& \left.\left.+\sum_{s=2}^{n}\left(p_{s}-1\right) x_{s}^{j}+(q-1) y^{j}\right]-\bar{R}\right\}-\sum_{j} \sum_{k \neq j} \lambda^{j k} v^{j k}
\end{aligned}
$$

The first-order conditions are:

$$
\frac{\partial \mathcal{L}}{\partial I^{j}}=\left(\gamma^{j}+\sum_{k \neq j} \lambda^{j k}\right) v_{I}^{j}-\pi^{j} \frac{\partial y^{j}}{\partial I^{j}} \phi^{\prime}+\mu \pi^{j}\left[1+\sum_{s=2}^{n}\left(p_{s}-1\right) \frac{\partial x_{s}^{j}}{\partial I^{j}}\right.
$$

\footnotetext{
${ }^{14}$ With one extra degree of freedom in setting commodity tax rates, $t_{1}$ is set equal to zero so that $p_{1}=1$.
} 


$$
\begin{aligned}
& \left.+(q-1) \frac{\partial y^{j}}{\partial I^{j}}\right]-\sum_{k \neq j} \lambda^{k j} v_{I}^{k j}=0, \quad j, k=1,2, \ldots, H, \\
\frac{\partial \mathcal{L}}{\partial c^{j}} & =\left(\gamma^{j}+\sum_{k \neq j} \lambda^{j k}\right) v_{c}^{j}-\pi^{j} \frac{\partial y^{j}}{\partial c^{j}} \phi^{\prime}+\mu \pi^{j}\left[-1+\sum_{s=2}^{n}\left(p_{s}-1\right) \frac{\partial x_{s}^{j}}{\partial c^{j}}\right. \\
& \left.+(q-1) \frac{\partial y^{j}}{\partial c^{j}}\right]-\sum_{k \neq j} \lambda^{k j} v_{c}^{k j}=0, \\
\frac{\partial \mathcal{L}}{\partial p_{i}} & =\sum_{j}\left(\gamma^{j}+\sum_{k \neq j} \lambda^{j k}\right) v_{i}^{j}-\left(\sum_{j} \pi^{j} \frac{\partial y^{j}}{\partial p_{i}}\right) \phi^{\prime}+\mu \sum_{j} \pi^{j}\left[\sum_{s=2}^{n}\left(p_{s}-1\right) \frac{\partial x_{s}^{j}}{\partial p_{i}}\right. \\
& \left.+x_{i}^{j}+(q-1) \frac{\partial y^{j}}{\partial p_{i}}\right]-\sum_{j} \sum_{k \neq j} \lambda^{j k} v_{i}^{j k}=0, \quad i=2, \ldots, n, \\
\frac{\partial \mathcal{L}}{\partial q} & =\sum_{j}\left(\gamma^{j}+\sum_{k \neq j} \lambda^{j k}\right) v_{q}^{j}-\left(\sum_{j} \pi^{j} \frac{\partial y^{j}}{\partial q}\right) \phi^{\prime}+\mu \sum_{j} \pi^{j}\left[\sum_{s=2}^{n}\left(p_{s}-1\right) \frac{\partial x_{s}^{j}}{\partial q}\right. \\
& \left.+y^{j}+(q-1) \frac{\partial y^{j}}{\partial q}\right]-\sum_{j} \sum_{k \neq j} \lambda^{j k} v_{q}^{j k}=0,
\end{aligned}
$$

where a subscript on $v$ denotes a partial derivative. Equations (24a)-(24d) characterize the Pareto-efficient allocations constrained both by self-selection as well as the linearity of commodity tax rates. The next two sections discuss the properties of the commodity tax rates and the general income tax function that can implement these allocations.

\section{Optimal commodity taxes}

Denote the compensated demand for a good by a "tilde" over the corresponding variable. In the Appendix, we prove that an interior solution satisfies the following conditions.

$$
\left(\begin{array}{c}
t_{y} \\
t_{2} \\
t_{3} \\
\vdots \\
t_{n}
\end{array}\right)=\boldsymbol{A}^{-1}\left(\begin{array}{l}
-\sum_{j} \sum_{k \neq j} \lambda^{k j}\left(y^{k j}-y^{j}\right) \frac{v_{c}^{k j}}{\mu} \\
-\sum_{j} \sum_{k \neq j} \lambda^{k j}\left(x_{2}^{k j}-x_{2}^{j}\right) \frac{v_{c}^{k j}}{\mu} \\
-\sum_{j} \sum_{k \neq j} \lambda^{k j}\left(x_{3}^{k j}-x_{3}^{j}\right) \frac{v_{c}^{k j}}{\mu} \\
\vdots \\
-\sum_{j} \sum_{k \neq j} \lambda^{k j}\left(x_{n}^{k j}-x_{n}^{j}\right) \frac{v_{c}^{k j}}{\mu}
\end{array}\right)+\left(\begin{array}{c}
1 \\
0 \\
0 \\
\vdots \\
0
\end{array}\right) \frac{\phi^{\prime}}{\mu}
$$


where

$$
\boldsymbol{A}=\left(\begin{array}{llllll}
\sum_{j} \pi^{j} \frac{\partial \tilde{y}^{j}}{\partial q} & \sum_{j} \pi^{j} \frac{\partial \tilde{y}^{j}}{\partial p_{2}} & \sum_{j} \pi^{j} \frac{\partial \tilde{y}^{j}}{\partial p_{3}} & \cdots & \sum_{j} \pi^{j} \frac{\partial \tilde{y}^{j}}{\partial p_{n}} \\
\sum_{j} \pi^{j} \frac{\partial \tilde{x}_{2}^{j}}{\partial q} & \sum_{j} \pi^{j} \frac{\partial \tilde{x}_{2}^{j}}{\partial p_{2}} & \sum_{j} \pi^{j} \frac{\partial \tilde{x}_{2}^{j}}{\partial p_{3}} & \cdots & \sum_{j} \pi^{j} \frac{\partial \tilde{x}_{2}^{j}}{\partial p_{n}} \\
\vdots & \vdots & \ddots & \vdots & \\
\sum_{j} \pi^{j} \frac{\partial \tilde{x}_{n}^{j}}{\partial q} & \sum_{j} \pi^{j} \frac{\partial \tilde{x}_{n}^{j}}{\partial p_{2}} & \sum_{j} \pi^{j} \frac{\partial \tilde{x}_{n}^{j}}{\partial p_{3}} & \cdots & \sum_{j} \pi^{j} \frac{\partial \tilde{x}_{n}^{j}}{\partial p_{n}}
\end{array}\right)
$$

Note that the right-hand side of (25) also depends on the tax rates so that we only have an implicit "solution" here. Nevertheless (25) is illuminating in a number of ways. First, it shows that the optimal tax on $y$ is not in general strictly Pigouvian. Again, given that the optimal tax rates on private goods are non-zero, this is to be expected. Second, the formula characterizing the tax on $y$ contains both Pigouvian and non-Pigouvian elements. The non-Pigouvian part reflects the binding self-selection constraints. Similar expressions appear in the characterization of optimal tax rates on private goods. Third, the formulas for taxation of $x_{i}^{j}$ 's and $y^{j}$ 's differ only in that the latter entails a Pigouvian term. Thus Sandmo's (1975) additivity property continues to hold in our setting for commodity taxes. ${ }^{15}$

Separable preferences and homogeneous tastes: Subsection 3.3.1, and Proposition 1, establish that if preferences are such that individuals of different types have identical marginal rates of substitution between every two consumer goods, then private goods should not be taxed and the tax on the externality generating good must be Pigouvian. While the result was formally derived for observable personal consumption levels, it continues to hold when the available information is on aggregate (as opposed to personal) consumption levels so that the available commodity tax instruments are linear. The point is that if nonlinear commodity taxes are not useful, linear commodity taxes cannot be either. Examination of (25) bears this out. Under this circumstance, from problem (17a)-(17b), $y^{k j}=y^{j}$ and $x_{s}^{k j}=x_{s}^{j}$ for all types $k$ and $j$, and all goods $s$.

\footnotetext{
${ }^{15}$ The system of equations in (25) also yield first-best taxes. Setting $\lambda^{j k}=0$ in these equations immediately result in $t_{i}$ 's $=0$ and $t_{y}=\phi^{\prime} / \mu$.
} 
One can easily see that, given these equalities, $t_{i}{ }^{\prime} \mathrm{s}=0$ and $t_{y}=\phi^{\prime} / \mu$ will be a solution to $(25)$.

\subsection{Independent demands}

To gain further insights into the structure of optimal taxes we next concentrate on the special case where there are no cross-price and income effects. It is easy to see from (25) that one will now have

$$
\begin{aligned}
t_{y} & =\frac{\sum_{j} \sum_{k \neq j} \lambda^{k j}\left(y^{k j}-y^{j}\right) v_{c}^{k j} / \mu}{-\sum_{j} \pi^{j} \partial \tilde{y}^{j} / \partial q}+\frac{\phi^{\prime}}{\mu}, \\
t_{i} & =\frac{\sum_{j} \sum_{k \neq j} \lambda^{k j}\left(x_{i}^{k j}-x_{i}^{j}\right) v_{c}^{k j} / \mu}{-\sum_{j} \pi^{j} \partial \tilde{x}_{i}^{j} / \partial p_{i}}, \quad i=2,3, \ldots, n .
\end{aligned}
$$

The formulas are strikingly simple, indicating a Pigouvian adjustment for $t_{y}$ in comparison to $t_{i}(i=2,3, \ldots, n)$. One may thus loosely say, "everything else equal", $t_{y}$ will exceed $t_{i}$ by the Pigouvian element. Note also that the non-Pigouvian element of $t_{y}$ and the tax on $t_{i}$ are identical in form. The denominators in these expressions can be written in terms of elasticities. They will then yield a generalization of the famous "inverse elasticity" rule in a setting with heterogeneous tastes and in the presence of a general income tax. These considerations apply both to $t_{y}$ and $t_{i}$

It is also interesting to note that the non-Pigouvian element of $t_{y}$ can take both positive as well as negative values so that $t_{y}$ can be greater as well as smaller than the Pigouvian tax. To see this, note that from the properties of the Slutsky matrix, the denominator of the non-Pigouvian element is positive. It then follows that the optimal tax on $y$ exceeds (is smaller than) the Pigouvian tax if $\sum_{j} \sum_{k \neq j}\left(y^{k j}-y^{j}\right) v_{c}^{k j} / \mu$ is positive (negative). A sufficient condition for this is that every mimicker, for whom the incentive compatibility constraint binds, would have a higher (lower) demand for $y$ than the type whom he mimics. This makes quite a bit of sense, as the tax would then hurt a mimicker more (less) in comparison to the person he mimics. Of course, the cited condition is only sufficient. Less stringent conditions may also do. 


\subsection{Uniform tax rates}

While optimal commodity tax rates are generally non-uniform, as a practical policy matter, almost all countries continue to levy indirect taxes that are, to a large extent, uniform. Often, this is motivated by political economy considerations. ${ }^{16}$ Whatever the cause, if this is the case, one must impose the uniformity as an additional constraint on the optimal tax problem of the government. The properties of the optimal tax rate on the externality generating good and the optimal income tax schedule will then have to be studied in the light of all the constraints imposed. The results of this section enable us to do this most easily. Imposing $t_{i}=0(i=1,2, \ldots, n)$ as an additional constraint on problem (19)-(21) does not change the structure of the remaining first-order conditions. The optimal tax on $t_{y}$ will then be found by setting $t_{i}=0$ in (A11) in the Appendix. The resulting formula for the optimal tax rate will then be seen to be identical to the one given by equation (27a). Our discussion in subsection 5.1 thus also applies here.

The following proposition summarizes the main results of this section.

Proposition 2 Assume aggregate (but not personal) consumption levels are publicly observable. Then

(i) the optimal (linear) tax rate on $y$ is characterized by both Pigouvian and nonPigouvian elements; while the externality leaves the formulas for the optimal tax rates on private goods unaffected. The optimal tax rates are characterized by (25)-(26).

(ii) Assume individuals of different types have identical marginal rates of substitution (at the same consumption bundle). Then (a) the optimal second-best tax on $y$ is Pigouvian, and (b) private goods must not be taxed.

(iii) Assume either income and cross-price effects are all zero, or that private goods must be taxed uniformly. Further assume that every mimicker, for whom the incentive

\footnotetext{
${ }^{16}$ This reflects many considerations. Differentiating amongst goods, that have no apparent differing characteristics, is open to criticisms on the basis of the principle of horizontal equity. Moreover, to the extent that differentiation may call for higher taxes on "necessities," its implementation may prove politically unpopular.
} 
compatibility constraint is binding, has a higher (lower) demand for $y$ than the type whom he mimics. Then the optimal tax on y exceeds (is smaller than) the Pigouvian tax.

\section{Income tax structure}

The last question for us to examine is to determine if the presence of externality affects the structure of the optimal income tax schedule, and if yes, how. Denote the general income tax schedule facing a $j$-type person by $T\left(I^{j}\right)$ so that his net income is $c^{j}=$ $I^{j}-T\left(I^{j}\right)$. The individual thus sets $-v_{I}^{j} / v_{c}^{j}=1-T^{\prime}\left(I^{j}\right)$ where we have assumed the tax function is differentiable with $T^{\prime}$ denoting the marginal income tax rate. ${ }^{17}$ Dividing equation (24a) by (24b), we can then immediately deduce that

$$
1-T^{\prime}\left(I^{j}\right)=\frac{1+\left[\sum_{i=2}^{n} \frac{\partial x_{i}^{j}}{\partial I^{j}} t_{i}+\frac{\partial y^{j}}{\partial I^{j}} t_{y}\right]-\frac{\phi^{\prime}}{\mu} \frac{\partial y^{j}}{\partial I^{j}}-\frac{\sum_{k \neq j} \lambda^{k j} v_{I}^{k j}}{\mu \pi^{j}}}{1-\left[\sum_{i=2}^{n} \frac{\partial x_{i}^{j}}{\partial c^{j}} t_{i}+\frac{\partial y^{j}}{\partial c^{j}} t_{y}\right]+\frac{\phi^{\prime}}{\mu} \frac{\partial y^{j}}{\partial c^{j}}+\frac{\sum_{k \neq j} \lambda^{k j} v_{c}^{k j}}{\mu \pi^{j}}},
$$

Equation (28) indicates that the marginal income tax rate is determined by three factors: incentive constraints, impacts of commodity taxes and the externality. The presence of the externality terms in (28) is quite telling. It shows that, with unobservable personal consumption levels, externality affects the shape of the income tax schedule. In particular, note that for the marginal income tax rate to be independent of the externality, one must set $t_{y}=\phi^{\prime} / \mu$. This, however, will be the case if conditions (12a)(12b) hold.

This result is in sharp contrast to the corresponding result in Section 3 where, with observable consumption levels, we found that externality did not affect the expressions for the marginal income tax rate. The intuition is simple. If $y^{j}$ 's are publicly observable, one can "directly" set them at the desired level (using a non-linear tax schedule to

\footnotetext{
${ }^{17}$ With a discrete distribution of types, the implementing tax function will generally be nondifferentiable at some points. There, we follow the standard terminology and continue to refer to $T^{\prime}\left(I^{j}\right) \equiv 1+v_{I}^{j} / v_{c}^{j}$ as the marginal income tax rate; see Stiglitz (1987).
} 
implement this level). The income tax instrument is not needed for this purpose. On the other hand, if $y^{j}$ 's are not publicly observable, they are "controlled" only indirectly. The income tax may then be used, in addition to the linear commodity tax on $y$, for this purpose. Specifically, the income tax affects $y^{j}$ 's and consequently this effect has to be taken into account in the design of the optimal income tax. (Any impact from income taxes on $y^{j}$ 's, when $y^{j}$ 's are observable, can be "neutralized" at no cost).

The foregoing result shows that Sandmo's additivity property, and Dixit's principle of targeting, does not hold in this setting. The reason for this is that the available direct instrument is more restrictive than the indirect instrument (a linear tax on the externality versus a nonlinear income tax). This is why in Section 3, where both instruments were nonlinear, the indirect instrument was not found useful. The same intuition applies to the findings of Sandmo (1975) and Bovenberg and van der Ploeg (1994) where all tax instruments (income as well as commodity) are linear and thus equally restrictive.

Equation (28) also sheds light on the direction of the impacts of externality and indirect tax terms on the marginal income tax rate. One can easily see that the normality of $y$ (so that $\partial y^{j} / \partial c^{j}>0$ ) and its complementarity to labor supply (so that $\partial y^{j} / \partial I^{j}>0$ ) tend to lower $T^{\prime}$, if $t_{y}>\phi^{\prime} / \mu$; and to increase it, if $t_{y}<\phi^{\prime} / \mu$. Similarly, the normality of $x_{i}\left(\partial x_{i}^{j} / \partial c^{j}>0\right)$ and its complementarity to labor supply $\left(\partial x_{i}^{j} / \partial I^{j}>0\right)$ tend to lower $T^{\prime}$, if $t_{i}>0$; and to increase it, if $t_{i}<0$. These implications will be reversed when the goods are inferior and/or substitutes to labor supply.

To derive a more precise characterization of the optimal income tax schedule, assume, as in Subsection 3.3, that there exists a category of persons, $h$, whom nobody mimics at equilibrium. This implies that no incentive constraint is binding towards this category, so that $\lambda^{k h}=0$ for all $k(\neq h)$. It will then immediately follow from (28) that

$$
1-T^{\prime}\left(I^{h}\right)=\frac{1+\left[\sum_{i=2}^{n} \frac{\partial x_{i}^{h}}{\partial I^{h}} t_{i}+\frac{\partial y^{h}}{\partial I^{h}} t_{y}\right]-\frac{\phi^{\prime}}{\mu} \frac{\partial y^{h}}{\partial I^{h}}}{1-\left[\sum_{i=2}^{n} \frac{\partial x_{i}^{h}}{\partial c^{h}} t_{i}+\frac{\partial y^{h}}{\partial c^{h}} t_{y}\right]+\frac{\phi^{\prime}}{\mu} \frac{\partial y^{h}}{\partial c^{h}}} .
$$

Now, as was shown in Subsection 5, if preferences are separable and tastes are homo- 
geneous, $t_{i}$ 's $=0$ and $t_{y}=\phi^{\prime} / \mu$, so that $T^{\prime}(h)=0$. However, in the absence of such restrictions on preferences, it is clear from (29) that in general

$$
T^{\prime}(h) \neq 0
$$

That is, the well-known "no distortion at the top" result no longer holds. However, it must be emphasized that the distortion does not arise because of the standard incentive constraints [equations (21)]. Its source is the "incomplete control" of consumption levels through linear commodity taxes (which arises because individual consumption levels are not observable). The point is that changes in net and gross income (labor supply) affect consumption levels. In turn, these changes affect welfare through tax revenues generated from commodity taxes and through the externality.

The marginal income tax rates of all other groups $(l \neq h)$, for whom some $\lambda^{k l}>0$, is found by substituting $l$ for $j$ in equation (28). The difference with $h$-group is that the marginal income tax rate facing $l$-types will also be affected by the incentive constraints. To shed more light on the properties of the marginal income tax rate, we consider the following special case.

\subsection{Uniform commodity tax rates}

With the uniformity of tax rates as an additional constraint, the marginal income tax rate is given by

$$
1-T^{\prime}\left(I^{j}\right)=\frac{1+\left(t_{y}-\frac{\phi^{\prime}}{\mu}\right) \frac{\partial y^{j}}{\partial I^{j}}-\frac{\sum_{k \neq j} \lambda^{k j} v_{I}^{k j}}{\mu \pi^{j}}}{1-\left(t_{y}-\frac{\phi^{\prime}}{\mu}\right) \frac{\partial y^{j}}{\partial c^{j}}+\frac{\sum_{k \neq j} \lambda^{k j} v_{c}^{k j}}{\mu \pi^{j}}} .
$$

This can be derived either directly or more simply by setting $t_{i}=0$ in (28).

Again differentiate between two groups of types: $h$ whom in equilibrium no person mimics and $l$ whom at least one type wants to mimic. For $h$ the incentive terms vanish. One can then see that the sign of $T^{\prime}\left(I^{h}\right)$ is identical to the sign of

$$
-\left(t_{y}-\frac{\phi^{\prime}}{\mu}\right)\left(\frac{\partial y^{h}}{\partial I^{h}}+\frac{\partial y^{h}}{\partial c^{h}}\right) .
$$


Consequently, $T^{\prime}\left(I^{h}\right)$ continues to be generally different from zero. Moreover, it may even be possible to sign $T^{\prime}\left(I^{h}\right)$. This depends on sign of $t_{y}-\phi^{\prime} / \mu$, which may be determined from (27a) and part (iii) of Proposition 2. It also depends on the sign of $\partial y^{h} / \partial I^{h}+\partial y^{h} / \partial c^{h}$. This latter term can be negative as well as positive. A sufficient condition for the former is for $y$ to be an inferior $\operatorname{good}\left(\partial y^{h} / \partial c^{h}<0\right)$ as well as a "substitute" to labor supply for $h$-type persons $\left(\partial y^{h} / \partial I^{h}<0\right)$. On the other hand, a sufficient condition for $\partial y^{h} / \partial I^{h}+\partial y^{h} / \partial c^{h}$ to be positive is that $y$ is a normal good $\left(\partial y^{h} / \partial c^{h}>0\right)$ and a "complement" to labor supply for $h$-type persons $\left(\partial y^{h} / \partial I^{h}>0\right)$.

The above factors continue to play the same role in determining the sign and the size of the marginal income tax rate facing $l$-types. However, for these persons, there will also be additional effects due to the incentive constraints along the familiar lines.

The following proposition summarizes the main results of this section.

Proposition 3 Assume aggregate (but not personal) consumption levels are publicly observable. Then

(i) in the absence of other restrictions, the externality affects the income tax structure;

(ii) the externality does not affect the income tax structure, if individuals of different types have identical marginal rates of substitution (at the same consumption bundle);

(iii) marginal income tax rate "at the top" will generally be nonzero; both positive and negative values are possible.

\section{Conclusion}

This paper has re-examined the Pigouvian prescription for correcting externalities in a second-best environment. It has also examined the properties of optimal commodity and income taxes in the presence of externalities. It has shown that the answers to these questions depend crucially on the structure of information in the economy, via its determining the type of tax instruments that are available to the government. 
Four main conclusions have emerged. First, environmental levies must generally consist of Pigouvian and non-Pigouvian terms and be nonlinear (if permitted by the informational structure). Second, externalities do not affect commodity tax formulas for private goods. This holds regardless of the public observability of individual consumption levels and thus applies to both linear and nonlinear commodity taxes. Third, externalities do not affect the income tax structure if commodity taxes are nonlinear and affect it if commodity taxes are linear. Fourth, a general income tax plus strictly Pigouvian taxes are sufficient for efficient taxation if individuals of different types have identical marginal rates of substitution (at any given consumption bundle).

An important implication of our second and third conclusions is that Sandmo's additivity property, and Dixit's Principle of targeting, breaks down when the tax instruments consist of linear commodity and nonlinear income taxes. In this case, while the tax formulas for private goods remain unaffected, income tax formulas change. The reason for this is that the available direct instrument is more restrictive than the (affected) indirect instrument (a linear tax on the externality versus a nonlinear income $\operatorname{tax})$. 


\section{Appendix}

Derivation of (25): Multiply equation (24b) by $y^{j}$, sum over $j$, add the resulting equation to (24d), and simplify. Then multiply (24b) by $x_{i}^{j}(i=2,3, \ldots, n)$, sum over $j$, add the resulting equation to (24c), and simplify. The following system of equation results

$$
\begin{aligned}
\sum_{j}\left(\gamma^{j}\right. & \left.+\sum_{k \neq j} \lambda^{j k}\right)\left(v_{q}^{j}+y^{j} v_{c}^{j}\right)-\phi^{\prime} \sum_{j} \pi^{j}\left(y^{j} \frac{\partial y^{j}}{\partial c^{j}}+\frac{\partial y^{j}}{\partial q}\right) \\
& +\mu \sum_{j} \pi^{j}\left[\sum_{s=2}^{n}\left(p_{s}-1\right)\left(y^{j} \frac{\partial x_{s}^{j}}{\partial c^{j}}+\frac{\partial x_{i}^{j}}{\partial q}\right)+(q-1)\left(y^{j} \frac{\partial y^{j}}{\partial c^{j}}+\frac{\partial y^{j}}{\partial q}\right)\right] \\
& -\sum_{j} \sum_{k \neq j} \lambda^{k j}\left(v_{q}^{k j}+y^{j} v_{c}^{k j}\right)=0, \\
\sum_{j}\left(\gamma^{j}\right. & \left.+\sum_{k \neq j} \lambda^{j k}\right)\left(v_{i}^{j}+x_{i}^{j} v_{c}^{j}\right)-\phi^{\prime} \sum_{j} \pi^{j}\left(x_{i}^{j} \frac{\partial y^{j}}{\partial c^{j}}+\frac{\partial y^{j}}{\partial p_{i}}\right) \\
& +\mu \sum_{j} \pi^{j}\left[\sum_{s=2}^{n}\left(p_{s}-1\right)\left(x_{i}^{j} \frac{\partial x_{s}^{j}}{\partial c^{j}}+\frac{\partial x_{s}^{j}}{\partial p_{i}}\right)+(q-1)\left(x_{i}^{j} \frac{\partial y^{j}}{\partial c^{j}}+\frac{\partial y^{j}}{\partial p_{i}}\right)\right] \\
& -\sum_{j} \sum_{k \neq j} \lambda^{k j}\left(v_{i}^{k j}+x_{i}^{j} v_{c}^{k j}\right)=0, \quad i=2,3, \ldots, n .
\end{aligned}
$$

Next make use of Roy's identity to set:

$$
\begin{aligned}
v_{q}^{j}+y^{j} v_{c}^{j} & =0, \\
v_{q}^{k j}+y^{k j} v_{c}^{k j} & =0, \\
v_{i}^{j}+x_{i}^{j} v_{c}^{j} & =0, \\
v_{i}^{k j}+x_{i}^{k j} v_{c}^{k j} & =0,
\end{aligned}
$$

and the Slutsky equation to write:

$$
\begin{aligned}
\frac{\partial y^{j}}{\partial q} & =\frac{\partial \tilde{y}^{j}}{\partial q}-y^{j} \frac{\partial y^{j}}{\partial c^{j}}, \\
\frac{\partial x_{s}^{j}}{\partial q} & =\frac{\partial \tilde{x}_{s}^{j}}{\partial q}-y^{j} \frac{\partial x_{s}^{j}}{\partial c^{j}},
\end{aligned}
$$




$$
\begin{aligned}
\frac{\partial y^{j}}{\partial p_{i}} & =\frac{\partial \tilde{y}^{j}}{\partial p_{i}}-x_{i}^{j} \frac{\partial y^{j}}{\partial c^{j}}, \\
\frac{\partial x_{s}^{j}}{\partial p_{i}} & =\frac{\partial \tilde{x}_{s}^{j}}{\partial p_{i}}-x_{i}^{j} \frac{\partial x_{s}^{j}}{\partial c^{j}} .
\end{aligned}
$$

Substituting from equations (A3)-(A6) and (A7)-(A10) in (A1)-(A2), making use of the symmetry of the Slutsky matrix, setting $p_{i}-1=t_{i}$ and $q-1=t_{y}$, upon further simplification and rearrangement, one arrives at

$$
\begin{aligned}
\left(\sum_{j} \pi^{j} \frac{\partial \tilde{y}^{j}}{\partial q}\right) t_{y} & +\sum_{i=2}^{n}\left(\sum_{j} \pi^{j} \frac{\partial \tilde{y}^{j}}{\partial p_{i}}\right) t_{i}= \\
& -\sum_{j} \sum_{k \neq j} \lambda^{k j}\left(y^{k j}-y^{j}\right) \frac{v_{c}^{k j}}{\mu}+\left(\sum_{j} \pi^{j} \frac{\partial \tilde{y}^{j}}{\partial q}\right) \frac{\phi^{\prime}}{\mu} \\
\left(\sum_{j} \pi^{j} \frac{\partial \tilde{x}_{s}^{j}}{\partial q}\right) t_{y} & +\sum_{i=2}^{n}\left(\sum_{j} \pi^{j} \frac{\partial \tilde{x}_{s}^{j}}{\partial p_{i}}\right) t_{i}= \\
& -\sum_{j} \sum_{k \neq j} \lambda^{k j}\left(x_{s}^{k j}-x_{s}^{j}\right) \frac{v_{c}^{k j}}{\mu}+\left(\sum_{j} \pi^{j} \frac{\partial \tilde{x}_{s}^{j}}{\partial q}\right) \frac{\phi^{\prime}}{\mu}, s=2, \ldots, n,
\end{aligned}
$$

Equations (A11)-(A12) are one way of characterizing the optimal commodity tax rates: $t_{i}$ 's and $t_{y}$.

To arrive at (25), use the definition of $\boldsymbol{A}$ in (26) to write out equations (A11)- (A12) in matrix notation:

$$
\boldsymbol{A}\left(\begin{array}{c}
t_{y} \\
t_{2} \\
t_{3} \\
\vdots \\
t_{n}
\end{array}\right)=\left(\begin{array}{l}
-\sum_{j} \sum_{k \neq j} \lambda^{k j}\left(y^{k j}-y^{j}\right) \frac{v_{c}^{k j}}{\mu}+\left(\sum_{j} \pi^{j} \frac{\partial \tilde{y}^{j}}{\partial q}\right) \frac{\phi^{\prime}}{\mu} \\
-\sum_{j} \sum_{k \neq j} \lambda^{k j}\left(x_{2}^{k j}-x_{2}^{j}\right) \frac{v_{c}^{k j}}{\mu}+\left(\sum_{j} \pi^{j} \frac{\partial \tilde{x}_{2}^{j}}{\partial q}\right) \frac{\phi^{\prime}}{\mu} \\
-\sum_{j} \sum_{k \neq j} \lambda^{k j}\left(x_{3}^{k j}-x_{3}^{j}\right) \frac{v_{c}^{k j}}{\mu}+\left(\sum_{j} \pi^{j} \frac{\partial \tilde{x}_{3}^{j}}{\partial q}\right) \frac{\phi^{\prime}}{\mu} \\
\vdots \\
-\sum_{j} \sum_{k \neq j} \lambda^{k j}\left(x_{n}^{k j}-x_{n}^{j}\right) \frac{v_{c}^{k j}}{\mu}+\left(\sum_{j} \pi^{j} \frac{\partial \tilde{x}_{n}^{j}}{\partial q}\right) \frac{\phi^{\prime}}{\mu}
\end{array}\right)
$$


Denote the first column vector of $\boldsymbol{A}$ by $\boldsymbol{a}_{1}$. Premultiplying (A13) by $\boldsymbol{A}^{-1}$ then yields

$$
\left(\begin{array}{c}
t_{y} \\
t_{2} \\
t_{3} \\
\vdots \\
t_{n}
\end{array}\right)=\boldsymbol{A}^{-1}\left(\begin{array}{l}
-\sum_{j} \sum_{k \neq j} \lambda^{k j}\left(y^{k j}-y^{j}\right) \frac{v_{c}^{k j}}{\mu} \\
-\sum_{j} \sum_{k \neq j} \lambda^{k j}\left(x_{2}^{k j}-x_{2}^{j}\right) \frac{v_{c}^{k j}}{\mu} \\
-\sum_{j} \sum_{k \neq j} \lambda^{k j}\left(x_{3}^{k j}-x_{3}^{j}\right) \frac{v_{c}^{k j}}{\mu} \\
\vdots \\
-\sum_{j} \sum_{k \neq j} \lambda^{k j}\left(x_{n}^{k j}-x_{n}^{j}\right) \frac{v_{c}^{k j}}{\mu}
\end{array}\right)+\left(\boldsymbol{A}^{-1} \boldsymbol{a}_{1}\right) \frac{\phi^{\prime}}{\mu},
$$

which is readily seen to be the system of equations (25) in the text. 


\section{References}

Atkinson, A.B. and J.E. Stiglitz (1976) "The design of tax structure: direct versus indirect taxation," Journal of Public Economics, 6, 55-75.

Atkinson, A.B. and J.E. Stiglitz (1980) Lectures on Public Economics. New-York: McGraw-Hill.

Bvenberg, L. and F. van der Ploeg (1994) "Environmental policy, public finance and the labor market in a second-best world," Journal of Public Economics, 55, 349-390.

Bvenberg, L. and L. Goulder (1996) "Optimal environmental taxation in the presence of other taxes: general equilibrium analyses," American Economic Review, 86, 985-1000.

Cremer, H., P. Pestieau and J-C. Rochet (1996) "Atkinson and Stiglitz proposition revisited: the case of a direct-indirect tax mix when there are two unobservables," mimeo.

Dixit, A. (1985) "Tax policy in open economies," in Handbook of Public Economics Vol 1, ed. Auerbach, A. and M. Feldstein. Amsterdam: North-Holland, 313-374.

Green, J. and E. Sheshinski (1976) "Direct versus indirect remedies for externalities," Journal of Political Economy, 84, 797-808.

Meade, J.E. (1952) "External economies and diseconomies in a competitive situation," Economic Journal, 62, 54-67.

Mirrlees, J.A. (1971) "An exploration in the theory of optimal income taxation," Review of Economic Studies, 28, 175-208.

Mirrlees, J.A. (1976) "Optimal tax theory: a synthesis," Journal of Public Economics, 6, 327-358.

Sandmo, A. (1975) "Optimal taxation in the presence of externalities," Swedish Journal of Economics, 77, 86-98.

Sandmo, A. (1976) "Direct versus indirect Pigovian taxation," European Economic Review, 7, 337-349.

Seade, J. (1977) "On the shape of optimal tax schedules," Journal of Public Economics, 7, 203-236.

Stiglitz, J.E. (1982) "Self-selection and Pareto efficient taxation," Journal of Public Economics, 17, 213-240.

Stiglitz, J.E. (1987) "Pareto efficient and optimal taxation and the new new welfare economics," in Handbook of Public Economics, Vol 2, ed. Auerbach, A. and M. Feldstein. Amsterdam: North-Holland, 991-1042. 
1 Introduction

1

intro 11 Page: 1

2 The model

$\begin{array}{llll}\text { se:model } & 2 & \text { Page: } & 4 \\ \text { eq:utility } & 1 & \text { Page: } & 5 \\ \text { eq:u2 } & 2 & \text { Page: } & 6\end{array}$

3 Observable individual purchases

se:personal

Page: $\quad 6$

3.1 Pareto-efficient allocations ... . .

$\begin{array}{llll}\text { eq:uj } & 3 \mathrm{a} & \text { Page: } & 6 \\ \text { eq:ujk } & 3 \mathrm{~b} & \text { Page: } & 6 \\ \text { eq:obj.g1 } & 4 & \text { Page: } & 7 \\ \text { eq:rc1 } & 5 & \text { Page: } & 7 \\ \text { eq:ssc1 } & 6 & \text { Page: } & 7 \\ \text { eq:lag1 } & 7 & \text { Page: } & 7 \\ \text { eq:lag2 } & 8 & \text { Page: } & 7 \\ \text { eq:lag2.dx } & 9 \mathrm{a} & \text { Page: } & 7 \\ \text { eq:lag2.dy } & 9 \mathrm{~b} & \text { Page: } & 8 \\ \text { eq:lag2.dI } & 9 \mathrm{c} & \text { Page: } & 8 \\ \text { eq:cg1 } & 10 \mathrm{a} & \text { Page: } & 8 \\ \text { eq:cg2 } & 10 \mathrm{~b} & \text { Page: } & 8 \\ \text { eq:cg3 } & 10 \mathrm{c} & \text { Page: } & 8\end{array}$

3.2 First-best taxation

\begin{tabular}{|c|c|c|}
\hline eq:fby & $11 \mathrm{a}$ & Page: \\
\hline eq: fbx & $11 b$ & Page: \\
\hline eq:fbI & $11 \mathrm{c}$ & Page: \\
\hline
\end{tabular}

3.3 Second-best taxation

...

$3.3 \quad$ Page: 9

3.3.1 Separable preferences and homogeneous tastes

$\begin{array}{llll}\text { se:nouse } & 3.3 .1 & \text { Page: } & 11 \\ \text { eq:as1 } & 12 \mathrm{a} & \text { Page: } & 11 \\ \text { eq:as2 } & 12 \mathrm{~b} & \text { Page: } & 12\end{array}$

3.3.2 A two-group illustration

............

eq: $u 2 g$

Page: 13

eq : $x 0$

14 Page: 13

eq:yl

15 Page: 13

eq:ylg

16a Page: 13

eq:yls

16b Page: 13

pr:personal

1 Page: 14

4 Observable aggregate purchases

se:aggregate

eq:ind.prob.obj

$4 \quad$ Page: 14

17a Page: 16 


eq:ind.prob.constr
eq:xij
eq:yj
eq: $v j$
eq: $x i j k$
eq:yjk
eq: $v j k$
eq: $V j$

$\begin{array}{lll}\text { 17b } & \text { Page: } & 16 \\ \text { 18a } & \text { Page: } & 16 \\ \text { 18b } & \text { Page: } & 16 \\ \text { 18c } & \text { Page: } & 16 \\ \text { 18d } & \text { Page: } & 16 \\ \text { 18e } & \text { Page: } & 16 \\ \text { 18f } & \text { Page: } & 16 \\ \text { 18g } & \text { Page: } & 16\end{array}$

4.1 Pareto-efficient (constrained) allocations . . . . . . . . . . . . . . . .

$\begin{array}{llll}\text { ss:pareto } & 4.1 & \text { Page: } & 17 \\ \text { eq:obj.g2 } & 19 & \text { Page: } & 17 \\ \text { eq:rc2 } & 20 & \text { Page: } & 17 \\ \text { eq:ssc2 } & 21 & \text { Page: } & 17 \\ \text { eq:lag1a } & 22 & \text { Page: } & 17 \\ \text { eq:lag2a } & 23 & \text { Page: } & 17 \\ \text { eq:lag2a.dI } & 24 \mathrm{a} & \text { Page: } & 18 \\ \text { eq:lag2a.dc } & 24 \mathrm{~b} & \text { Page: } & 18 \\ \text { eq:lag2a.dp } & 24 \mathrm{c} & \text { Page: } & 18 \\ \text { eq:lag2a.dq } & 24 \mathrm{~d} & \text { Page: } & 18\end{array}$

5 Optimal commodity taxes

$\begin{array}{llll}\text { se:commodity } & 5 & \text { Page: } & 18 \\ \text { eq:tv } & 25 & \text { Page: } & 18 \\ \text { eq:matrix.def } & 26 & \text { Page: } & 19 \\ \text { se:commodity1 } & 5 & \text { Page: } & 19\end{array}$

5.1 Independent demand

..........

$\begin{array}{llll}\text { se: ind } & 5.1 & \text { Page: } & 20 \\ \text { eq:ty.ind } & 27 \mathrm{a} & \text { Page: } & 20 \\ \text { eq:ti.ind } & 27 \mathrm{~b} & \text { Page: } & 20\end{array}$

5.2 Uniform tax rates . . . . . . . . . . . . . . . . . . . 21 pr:aggcon $\quad 2 \quad$ Page: 21

6 Income tax structure

$\begin{array}{llll}\text { se:income } & 6 & \text { Page: } & 22 \\ \text { eq:mitr1 } & 28 & \text { Page: } & 22 \\ \text { eq:mitrh0 } & 29 & \text { Page: } & 23\end{array}$

6.1 Uniform commodity tax rates ..................... . . . . . . .

$\begin{array}{llll}\text { eq: } \text { mitr2 } & 30 & \text { Page: } & 24 \\ \text { pr:income } & 3 & \text { Page: } & 25\end{array}$

7 Conclusion

$\begin{array}{llll}\text { se:conclus } & 7 & \text { Page: } & 25 \\ \text { eq:opq1 } & \text { A1 } & \text { Page: } & 27 \\ \text { eq:opi1 } & \text { A2 } & \text { Page: } & 27 \\ \text { eq: royyj } & \text { A3 } & \text { Page: } & 27\end{array}$




eq:royykj
eq:royxj
eq:royxkj
eq:slyy
eq:slxy
eq:slyx
eq:slxx
eq:opq2
eq:opp2
eq:matrix1
matrix2

A4 Page: 27

A5 Page: 27

A6 Page: 27

A7 Page: 27

A8 Page: 27

A9 Page: 28

A10 Page: 28

A11 Page: 28

A12 Page: 28

A13 Page: 28

A14 Page: 29 